$\operatorname{Cong}-930269--20$

UCRL-JC-112247

PREPRINT

\title{
Longitudinal Beam Dynamics for Heavy Ion Fusion Using WARPrz
}

Debra A. Callahan, A. Bruce Langdon, Alex Friedman, and Irving Haber

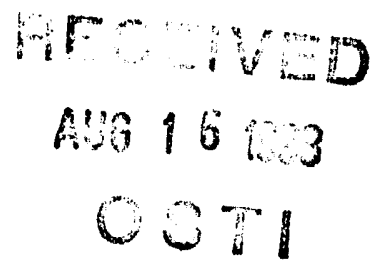

This paper was prepared for submittal to the Proceedings of the Computational Accelerator Physics Conference Pleasanton, CA

February 22-26, 1993

February 22, 1993

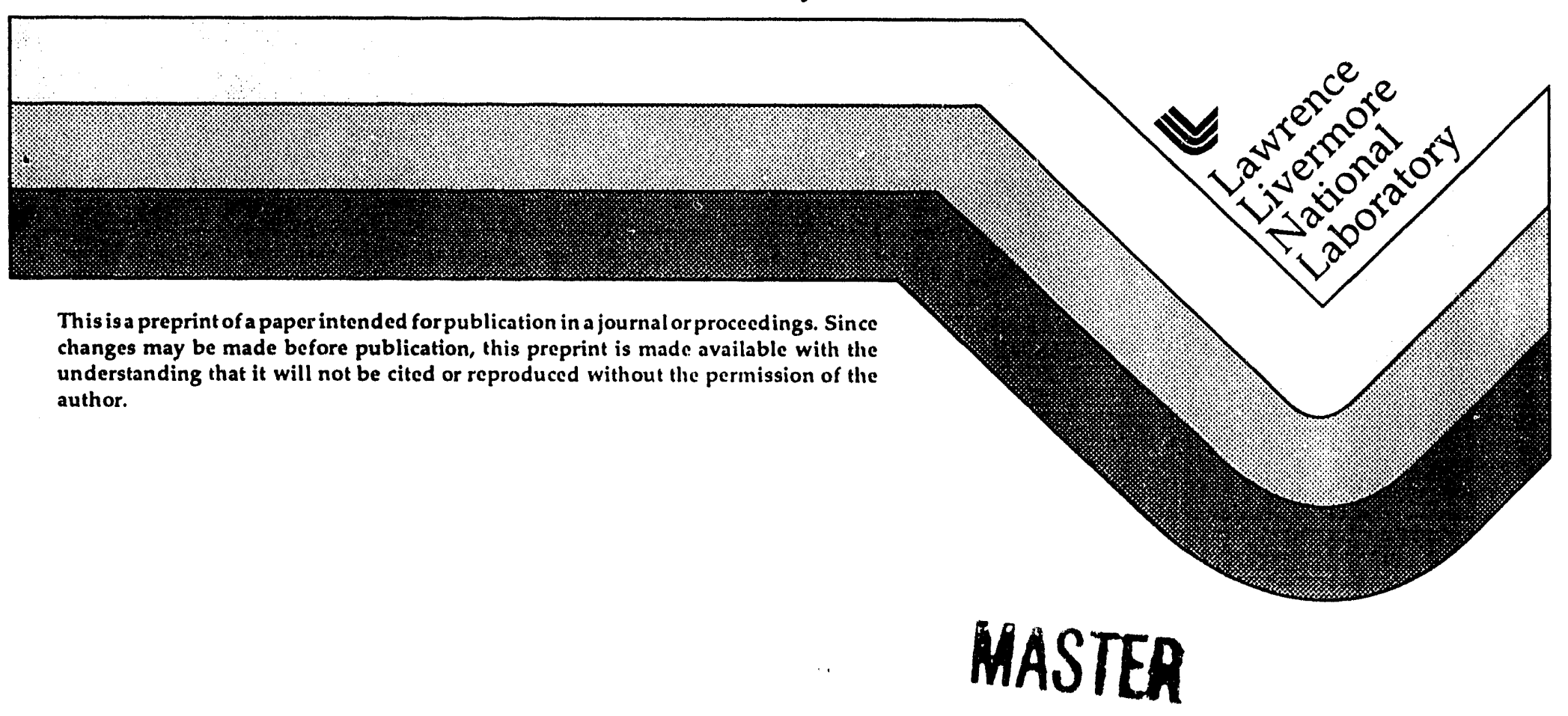




\section{DISCLAIMER}

This document was prepared as an account of work sponsored by an agency of the United States Government. Neither the United States Government nor the University of Callfornia nor any of their employees, makes any warranty, express or implied, or assumes any legal liability or responsibility for the accuracy, completeness, or usefulness of any information, apparatus, product, or process completeness, or usefulness of any information, apparatus, product, or process
disclosed, or represents that its use would not infinge privately owned rights. Reference herein to any specific commercial product, process, or service by trade name, trademark, manufacturer, or otherwise, does not necessarily constitute or imply its endorsement, recommendation, or favoring by the United States Government or the University of California. The views and opinions of authors expressed herein do not necessarily state or reflect those of the United States Government or the University of California, and shall not be used for advertising or product endorsement purposes. 


\title{
LONGITUDINAL BEAM DYNAMICS FOR HEAVY ION FUSION USING WARPRZ*
}

\author{
Debra A. Callahan, A. Bruce Langdon, Alex Friedman \\ Lawrence Livermore National Laboratory, Livermore CA 94550 \\ Irving Haber \\ Naval Research Laboratory, Washington DC 20375
}

\begin{abstract}
WARPrz is a 2.5 dimensional, cylindrically symmetric, electrostatic, particlein-cell code. It is part of the WARP family of codes which has been developed to study heavy ion fusion driver issues. WARPrz is being used to study the longitudinal dynamics of heavy ion beams including a longitudinal instability that is driven by the impedance of the LINAC accelerating modules. This instability is of concern because it can enhance longitudinal momentum spread; chromatic abhoration in the lens system restricts the amount of momentum spread allowed in the beam in the final focusing system. The impedance of the modules is modeled by a continuum of resistors and capacitors in parallel in WARPrz. We discuss simulations of this instability including the effect of finite temperature and reflection of perturbations off the beam ends. We also discuss intermittency of axial confining fields ("ears" fields) as a seed for this instability.
\end{abstract}

\section{INTRODUCTION}

Because of the large costs involved in building a full scale heavy ion fusion (HIF) accelerator, much effort has gone into simulating the physics of space charge dominated beams needed for HIF. These simulations have been coupled with experiments when possible. The WARP family of $\operatorname{codes}^{1}$ has been developed to study driver issues. The code is made up of five major physics packages: a $3 \mathrm{~d}$ particle-in-cell code in Cartesian geometry, a $3 \mathrm{~d}$ electrostatic field solver, a cylindrically symmetric $(r, z)$ particle-in-cell code, an $r, z$ electrostatic field solver, and an envelope code. This family of codes is being used to study a variety of heavy ion fusion issues. ${ }^{2,3}$

The longitudinal dynamics of the beams in the induction linear accelerator for heavy ion fusion have been of concern for some time. Since we must focus the beams onto the target at the end of the driver, we cannot tolerate a large spread in longitudinal momentum. The longitudinal wall impedance instability can cause small errors launched at the beam head to grow to an unacceptable size. This instability has the same mechanism used in "resistive wall" amplifiers with the impedance coming from the accelerating modules.

In order to model the longitudinal dynamics of these beams, the $r, z$ portion of the WARP code was developed. This code is a 2.5 dimensional, cylindrically symmetric particle-in-cell code. Field solution calculations are done in a window that moves with the beam. In this window, the fields are very close to purely electrostatic since the force due to magnetic fields is down by $(u / c)^{2}$ from the force due to the electric fields where $u$ is the velocity in the beam frame. The beam frame velocity for a heavy ion fusion driver is much less than $1 \%$ of the speed of light.

*This work was preformed under the auspices of the U.S. D.O.E. by Lawrence Livermore National Laboratory under contract W-7405-ENG-48, and by the Naval Research Laboratory under contracts DE-AI05-92ER54177 and DE-AI05-83ER40112. 


\section{LONGITUDINAL INSTABILITY}

The longitudinal wall impedance instability can be seen via a simple fluid model. If we consider an incompressible beam with radius $a$ traveling down a pipe of radius $r_{\text {wall }}, 1-d$ linear cold fluid theory shows that two waves will developa forward traveling wave and a backward traveling wave. These waves propagate with a phase velocity in the beam frame given by

$$
v_{\text {phase }}=\sqrt{\frac{Z e \lambda g}{4 \pi \epsilon_{0} m}}
$$

where $Z$ is the charge, $\lambda$ is the line charge density (with units of charge/length), $\mathrm{m}$ is the mass, $g=\ln \left(r_{\text {wall }}^{2} / a^{2}\right)$. Adding a wall composed of a continuum of resistors and capacitors in parallel to this calculation results in the forward traveling wave decaying while the backward traveling wave grows. This growth is largest when the perturbation wavelength is large compared with the pipe radius. In a heavy ion fusion driver, the impedance that drives this instability comes from the induction acceleration modules.

In order to study the longitudinal instability, we added a model for a wall with a continuum of resistors and capacitors in parallel ${ }^{4,5}$ to WARPrz. This approximation for the induction modules contains the relevant physics and also corresponds well with much of the analytic work being done. We calculate the resistive wall contribution to the electric field using the Poisson solve at the boundary. This is smoother and more physical than using the explicit beam current.

Our model assumes a continuity equation for the wall surface charge, $\sigma$ which has units of charge/area.

$$
\frac{\partial \sigma}{\partial t}+\frac{\partial K_{z}}{\partial z}=0
$$

where $K_{z}$ is the surface current. We use Ohm's law for a resistor and capacitor in parallel

$$
2 \pi r_{\text {wall }} \eta K_{z}=E_{z}+\eta C \frac{d E_{z}}{d t}
$$

where $\eta$ is the resistance per unit length and $\eta C$ is the "RC" time. Combining these equations and using the definition of the electrostatic potential gives

$$
\frac{\partial \sigma}{\partial t}=\frac{1}{2 \pi r_{\text {wall }}} \frac{\partial}{\partial z}\left[\frac{1}{\eta}\left(\frac{\partial \phi}{\partial z}+\eta C \frac{d}{d t} \frac{\partial \phi}{\partial z}\right)\right]
$$

For a constant $\eta$ and $\eta C$, we can Fourier transform with respect to $z$ and use a finite difference approximation to the time derivatives. This then becomes the boundary condition for our Poisson solve routine. We also assume the radial electric field is zero outside the pipe wall. Since our compltation mesh is a window moving with the beam, we need to advect the surface charge density and electrostatic potential backward when the mesh moves forward. This method has been tested in WARPrz by running in the linear, cold beam regime and the growth rates agree with the 1-d fluid theory to within $1.5 \%$.

Our first set of realistic simulations included only a resistive component to the impedance. When resistance is turned on, we need to add an external electric field that keeps the beam from losing all of its energy to the wall. The field that we added was simply

$$
E_{z, \text { external }}(z)=\eta I_{0}(z),
$$


where $\eta$ is the resistance per unit length (ohms/meter) and $I_{0}(z)$ is the current profile at time zero. Since the axial electric field does not vary much across the beam radius, we applied this same external field at all radii.

\begin{tabular}{|l|r|}
\hline Beam velocity & $1 / 3 \mathrm{c}$ \\
\hline Beam current & $3000 \mathrm{Amps}$ \\
\hline Pulse length & 10 meters \\
\hline Beam radius/Pipe radius & .4 \\
\hline Perpendicular temperature & $10 \mathrm{keV}$ \\
\hline Parallel temperature & $10 \mathrm{keV}$ \\
\hline
\end{tabular}

Table 1: Beam parameters near the end of a HIF accelerator

We found that slight mismatches in the external field caused perturbations to be launched from the beam head and tail. Table 1 shows the parameters for the simulation; these are similar to those proposed for the end of the acceleration section of a HIF driver. These parameters were chosen because the growth rate for the longitudinal instability is the largest in this regime. The wall resistance was $100 \Omega / \mathrm{m}$ with no capacitance. Figure 1 shows the perturbation that has been launched from the beam head (right hand side of the plots) in the electrostatic potential on axis vs $z$ and the $z-v_{z}$ phase space at time $4.9 \mu \mathrm{s}$. At this time, the perturbation can be seen as a shallow dip the potential about 2.5 meters from the beam head. Figure 2 shows the same plots at time $17 \mu \mathrm{s}$. Growth is readily seen. The growth rate measured in this case is about $15 \%$ smaller than the cold beam theory predicts.

Electrostatic Potential on Axis vs $z$

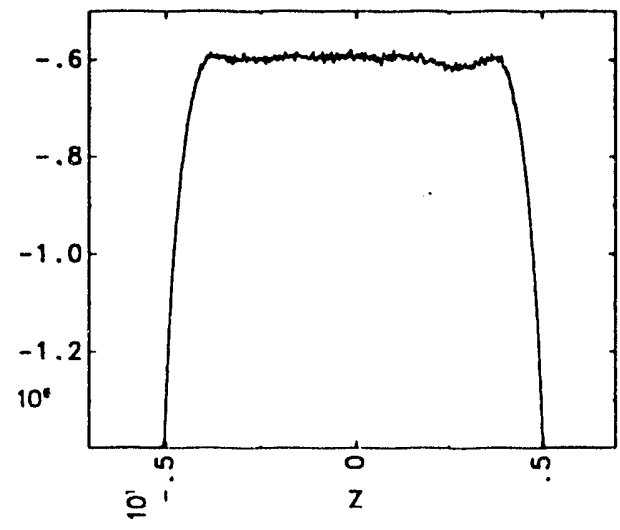

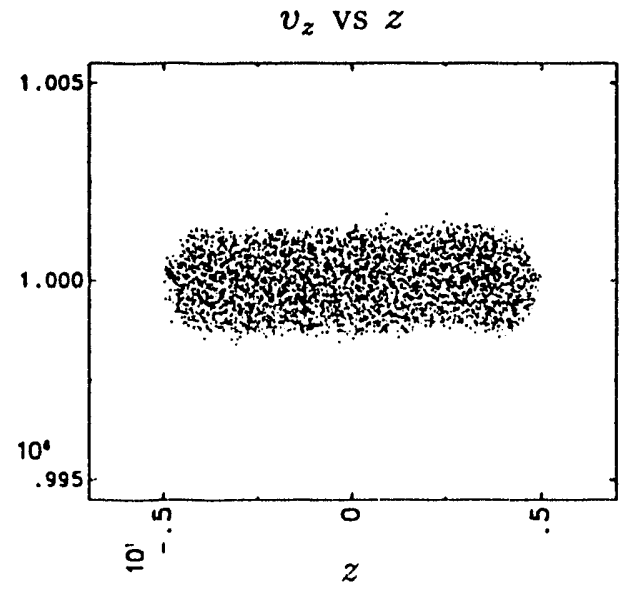

Figure 1: A perturbation is launched from the beam head

Continuing this simulation, we see that the perturbation reaches the beam tail and reflects, and begins moving towards the head. After reflection, the perturbation is seen to decay as is predicted by the cold beam theory. We do notice, however, that during reflection the perturbation width narrows. We believe this is a nonlinear effect since it has been observed to be more severe in larger perturbations.

The cold beam theory shows that capacitance has a partially stabilizing effect on this instability. When capacitance is added to the system, the external field 
Electrostatic Potential on Axis vs $z$

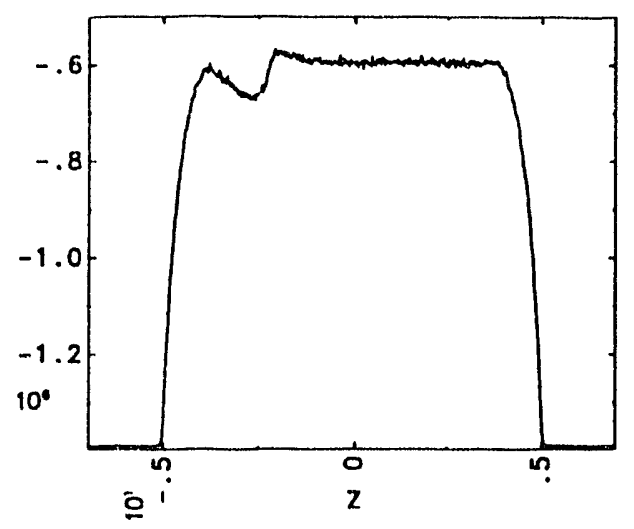

$v_{z}$ VS $z$

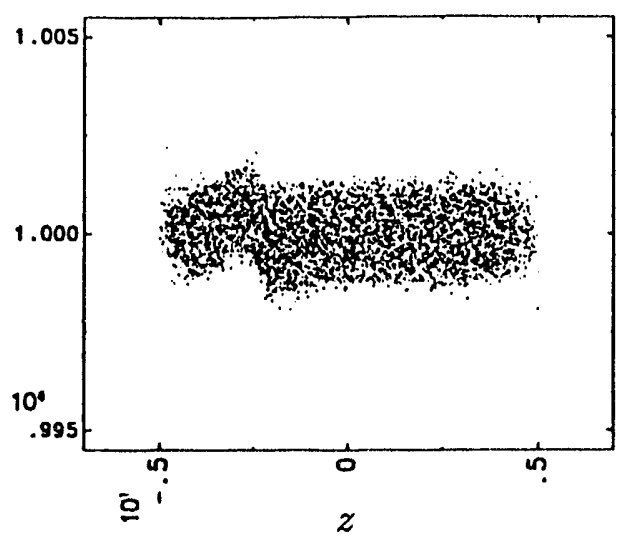

Figure 2: The perturbation grows as it travels from beam head to tail

added to keep the beam from losing its energy to the wall becomes:

$$
\eta C \frac{d E_{z, \text { external }}}{d t}+E_{z, \text { external }}=\eta I_{0}(z)
$$

We found when we did simulations with $\eta=100 \Omega / m$ and $\eta C=2.0 \times 10^{-8}$ seconds that very long wavelength perturbations were introduced because the equilibrium was not perfect. These perturbations did not undergo much growth, however. Video animation of the simulation showed that the perturbation sloshed back and forth from beam head to tail with little change in size.

\section{INTERMITTENTLY APPLIED “EAR” FIELDS}

To get a realistic look at the effects of the longitudinal instability, we need to look for sources of errors that will generate perturbations on the beam in an actual device. One source of such perturbations is the intermittently applied axial confining electric fields ("ear" fields). In the simulations in the previous section, we applied ear fields at each time step and these fields were designed to keep the beam from expanding or contracting. In an experiment, these fields will be applied at fixed locations along the accelerator and the beam will expand and contract between applications. The application of these fields can cause a train of perturbations to be launched from the beam head and these perturbations will be amplified by the longitudinal instability. steps:

Each application of the ears has a period $\left(\tau_{\text {ears }}\right)$ made up of the following

1. Let the beam expand from time 0 to $\tau_{\text {off }} / 2$.

2. At time $\tau_{\text {off }} / 2$, turn the ears on for a time $\tau_{\text {on }}$ and compress the beam.

3. At time $\tau_{\text {off }} / 2+\tau_{\text {on }}$, turn the ears off and let the beam expand until time $\tau_{\text {ears }}=\tau_{\text {on }}+\tau_{\text {off }}$. At this time, the beam should be back to its original length.

The waveform for the electric ears field was obtained by applying a force proportional to the integral over time of the force that was present during the 
free expansion.

$$
E_{z}(z)=F(z) / q=-\hat{F} \int_{0}^{\tau_{\text {on }}} \frac{m}{q} \frac{d u(z, t)}{d t} d t=-\hat{F} \frac{m}{q} u\left(z, \tau_{\text {on }}\right)
$$

where $u(z, t)$ is the average particle velocity as a function of $z$ in the beam frame, $m$ is the mass, and $q$ is the charge. In evaluating the integral, we have assumed that the average velocity in the beam frame is zero at time zero. The proportionality constant $\hat{F}$ should be approximately equal to $2 / \tau_{\text {on }}$ where the factor of two comes in because we need to not only reverse the expansion but also contract the beam so that it will expand back to its original size after the second $\tau_{\text {off }} / 2$ of expansion time. In practice, we start with this value and then adjust $\hat{F}$ until we get good results from one ears application.

Since we are not accelerating the beam, we chose accelerator parameters like those near the end of the acceleration section of the HIF driver because the growth rate for the longitudinal instability is the largest there. In fact, the beam will not spend much time in this section of the machine, so we are looking at a worst-case scenario. The simulation parameters are summarized in table 1 . This simulation had a wall resistance of $100 \mathrm{ohms} /$ meter with no capacitance. The schedule for the ears was $\tau_{\text {off }}=1 \mu s$ (or 100 meters at $v_{\text {beam }}=c / 3$ ) and $\tau_{\text {on }}=.1 \mu s$.

The first simulation in this series was an attempt to apply the intermittent ears with as little damage to the beam as possible ("perfect" ears). Figure 3 shows the electrostatic potential on axis vs axial position after 14 and 22 applications of the ears. By 14 applications, the perturbation launched at the beam head (the right hand side of the beam in the plots) has traveled about 7 meters and can be seen as a broad, shallow dip in the potential. After 22 applications, the beam looks similar. In this run, we do not see a train of perturbations being launched off the beam head and growing towards the tail. The beam remains very smooth.

\section{Electrostatic Potential on Axis vs $z$}

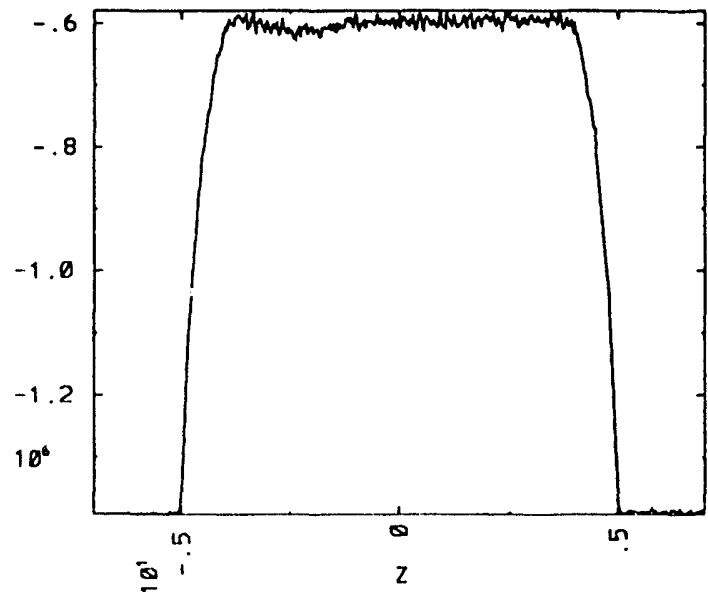

14 applications

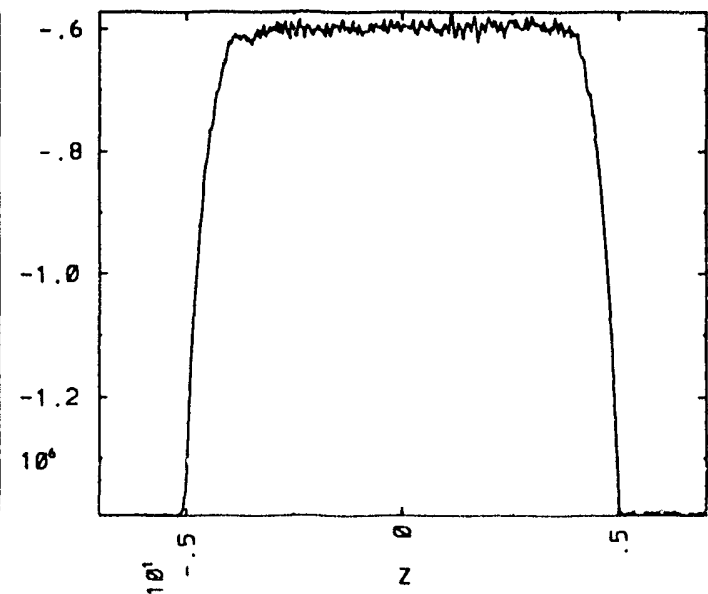

22 applications

Figure 3: Applying "perfect" ears

The electric field that we are applying in this case is fairly large. Figure $4 a$ shows the applied ear field as a function of $z$. The maximum electric field needed to contain the beam is on the order of $17.5 \mathrm{MV} / \mathrm{m}$. This is about three times 
the size of the field that we would like to apply in the experiment and tells us that at the end of the accelerator, we will need to apply the ears more frequently than every 100 meters. It is comforting, however, to see that the beam remains in good shape even if we apply the ears as infrequently as 100 meters.

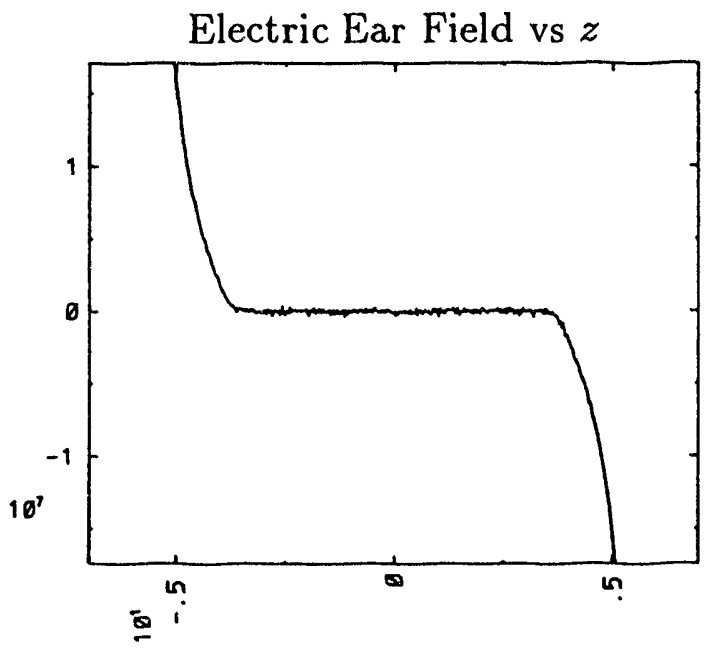

a) Ear Field (V/m)

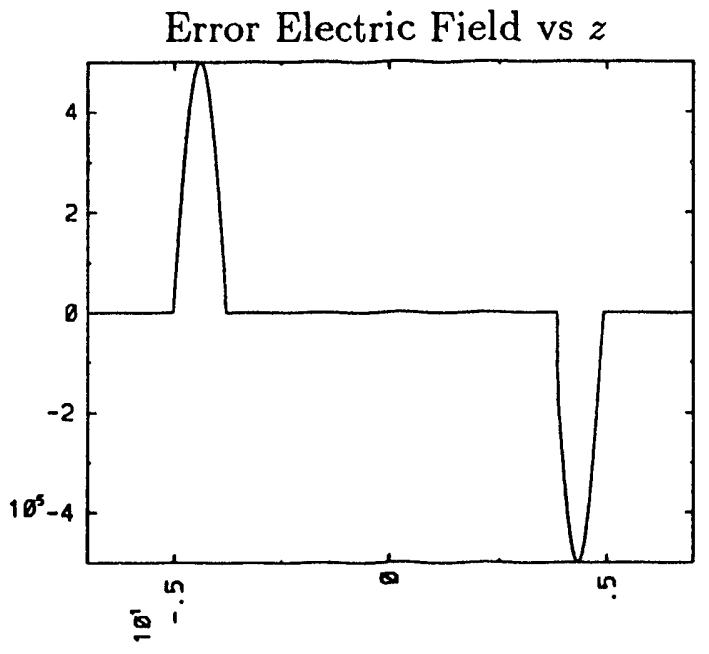

b) Error added to Ear field (V/m)

Figure 4: Ear field and error introduced

Our first attempt at studying imperfect ears was to add a "bump" to the ear fields used in the case of perfect ears. Figure $4 \mathrm{~b}$ shows the error added to the ears. The bump had the algebraic form of one half the period of a sine wave and magnitude of $5 \%$ of the local ear field. This bump has the effect of making the ears too large. We believed that by applying an error in the same direction every time, we would see a worst case because there was no way for the errors to cancel one another out. We found that this was not the case.

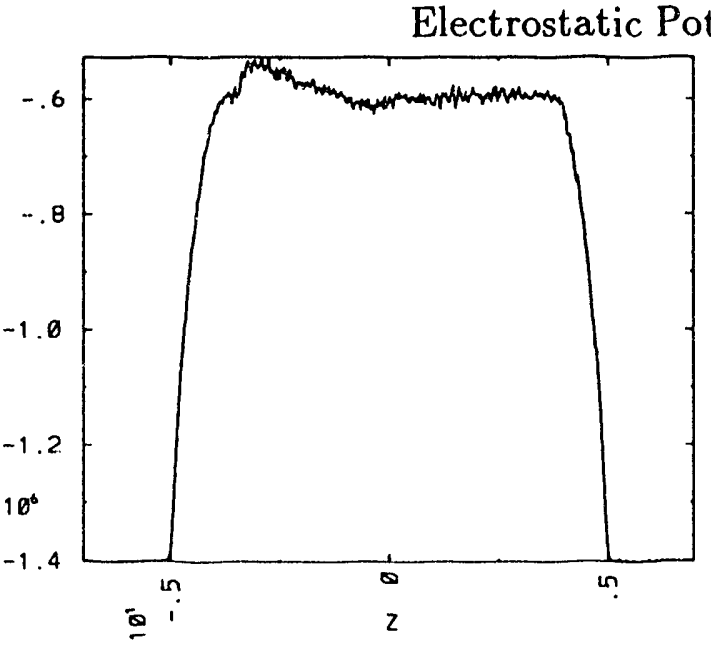

14 applications

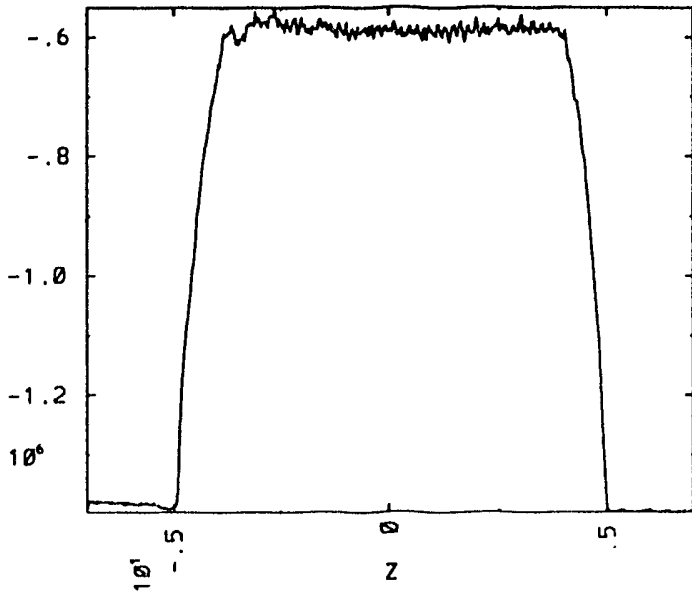

22 applications

Figure 5: Applying ears that are always too large

When we applied these ears, we found that the first few applications caused a perturbation to be launched from the beam head. Figure 5 shows this perturbation after 14 applications as it nears the beam tail after undergoing amplification 
by the longitudinal instability. Interestingly, if we look after 22 applications, we do not see a trail of perturbations coming off the beam head as we expect. In fact, the beam has adjusted itself to the error in the ears.

This phenomenon has also been seen in experiments done by A. Faltens. These experiments were designed to test longitudinal bunch control in the beam tail on the SBTE at LBL. In this experiments, no attempt was made to match the waveform of the applied ear fields to the beam profile. Instead, fields of the form $[1-\exp (-a t)]$ were applied. In the experiment, mismatches in the ear fields caused waves to be launched from the beam tail in the early pulsers, but at later times the beam reached a new steady state configuration.

Once we found that the beam could adjust to a systematic error in the ears, we tried alternating the error. In this simulation, we applied the same size and shape error as in the previous run, but we alternated the sign of the error with each application. This amounted to applying ears that were too large on one application followed by ears that were too small on the next application.

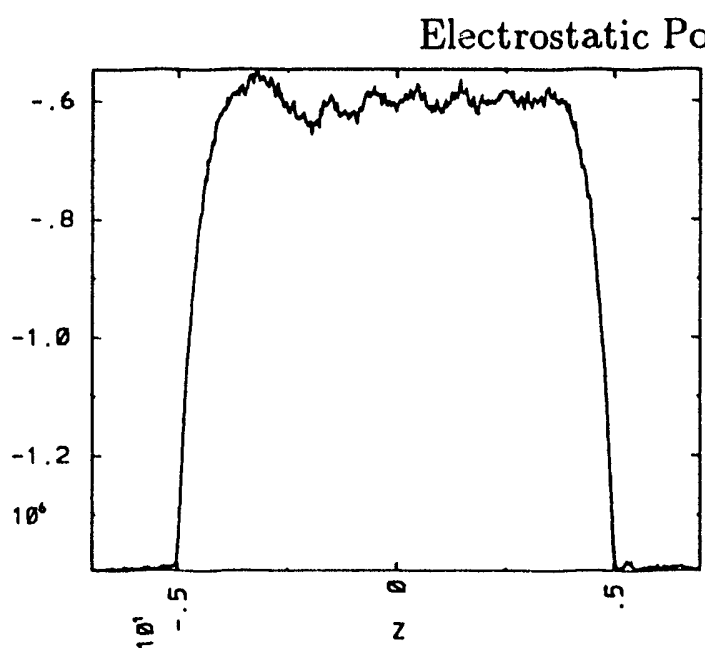

14 applications

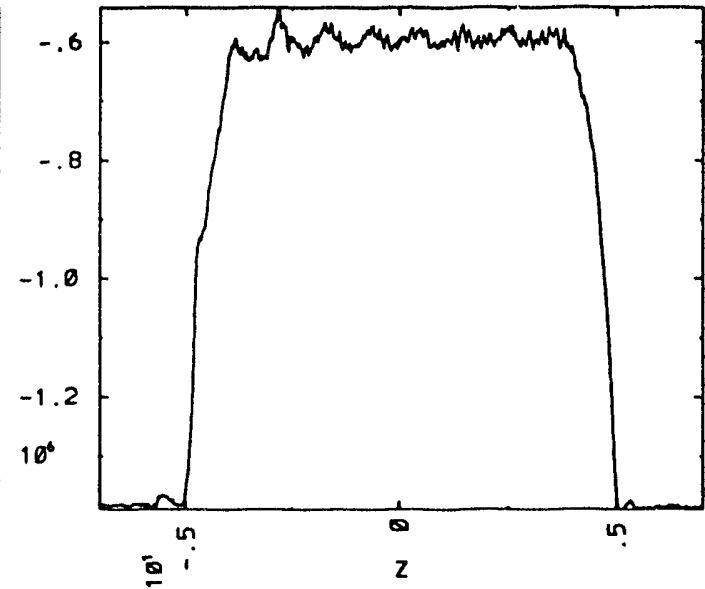

22 applications

Figure 6: Applying ears that alternate too large and too small

As figure 6 shows, we generated the expected train of perturbations coming off the beam head and moving towards the tail. We expect a similar train of perturbations coming from the beam tail, but these perturbations decay as they travel towards the beam head and are not seen. The wavelength of the perturbations is measured to be about 1 meter. We calculate $2 \tau_{\text {ears }} v_{\text {phase }}=1.07$ meters. This would indicate the cycle is:

1. hit the beam too hard and cause a enhancement region

2. this region moves away from the beam head at the wave velocity ( $\left.v_{\text {phase }}\right)$ in the time between ear applications

3. hit the beam too softly and cause a depletion region

4. this region moves away from the beam head at the wave velocity $\left(v_{\text {phase }}\right)$ in the time between ear applications

5. repeat 
Such a cycle implies a perturbation wavelength of $2 \tau_{\text {ears }} v_{\text {phase }}$ which is the observed wavelength.

In the future, we will study further aspects of longitudinal beam dynamics for HIF with WARPrz. This will include extending the work presented here to cooler beams and studying other sources of beam perturbations such as errors in the accelerating fields. Since our beam travels at speeds much slower than the speed of light, it is possible to detect beam perturbations, send a signal down the accelerator, and correct the errors downstream. Such "feed-forward" schemes have been demonstrated in 1-d simulations ${ }^{6}$ and we would like to further explore these ideas with WARPrz. We are also interested in how the beam emittance varies with longitudinal position in the beam ends in an equilibrium state.

\section{CONCLUSIONS}

We have modeled the longitudinal dynamics of space charge dominated heavy ion beams for heavy ion fusion. We have included a model of the impedance due to the induction acceleration modules as a continuum of resistors and capacitors in parallel in the WARPrz code. Using this code, we have modeled the longitudinal instability and seen waves launched from the beam head grow as they travel toward the tail, reflect off the beam tail, and decay as they travel forward. We have seen the partially stabilizing effect of the capacitive component of the impedance. We have looked at intermittently applying axial confining fields ("ear fields") as a seed for this instability. We have seen that the beam can adjust to systematic errors in the ear fields; however more random errors, such as errors which alternate sign, generate a train of perturbations on the beam.

\section{REFERENCES}

[1] A. Friedman, D. A. Callahan, D. P. Grote, A. B. Langdon, and I. Haber, Proc. of the Conference on Computer Codes and the Linear Accelerator Community, Los Alamos, NM, Jan 1990.

[2] A. Friedman, et. al. this meeting.

[3] D. P. Grote, et. al. this meeting.

[4] D. A. Callahan, A. B. Langdon, A. Friedman, D. P. Grote, and I. Haber, Particle Accelerators, 37-8, 97, (1992).

[5] D. A. Callahan, A. B. Langdon, A. Friedman, D. P. Grote, and I. Haber, Proc. of the International Conference on the Numerical Simulations of Plasmas, Annapolis, MD, 1991.

[6] K. Hahn, Bul. Am. Phys. Soc., Seattle, WA, 1992. 

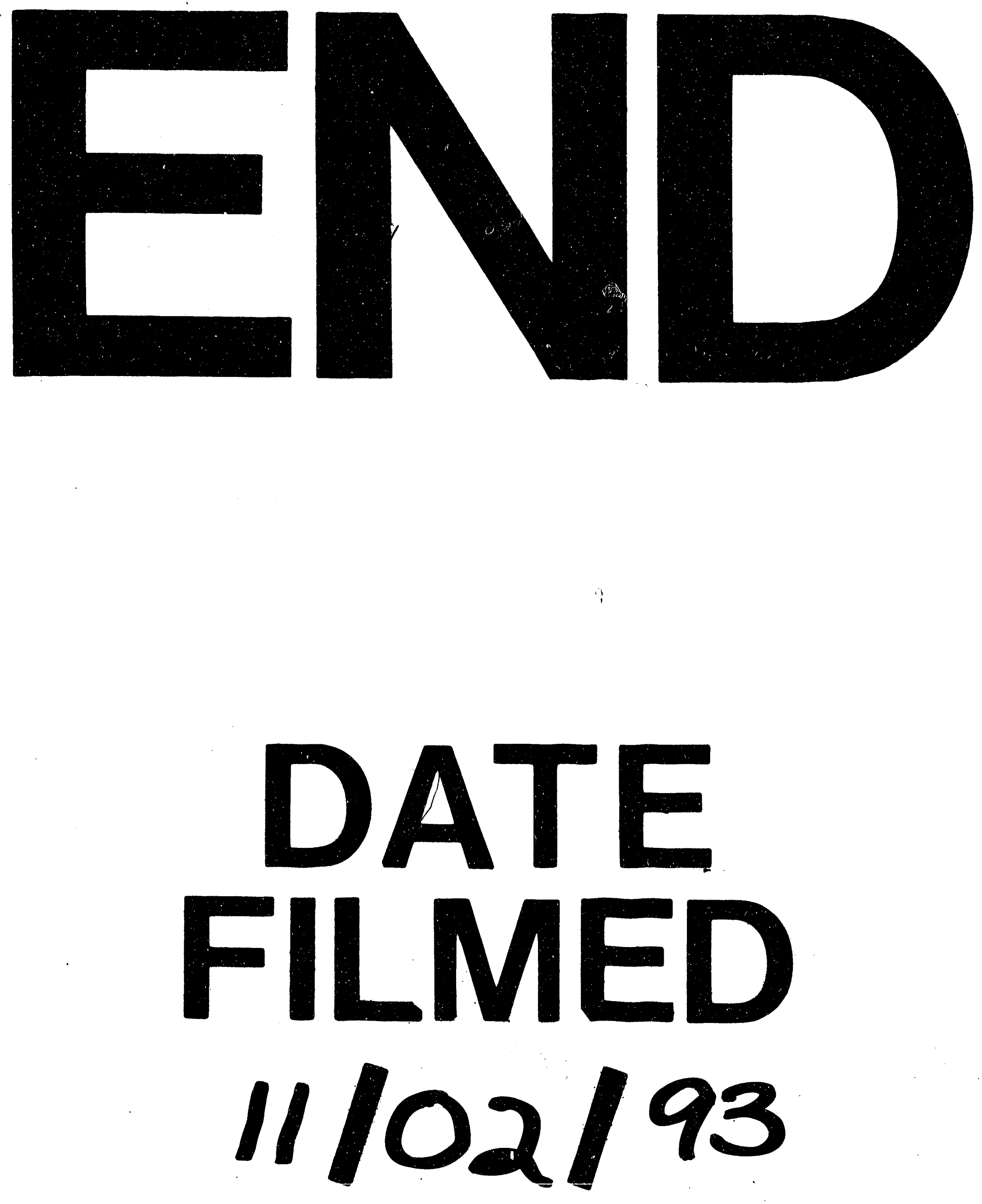

1 
